$$
\text { "antibi" — 2007/8/17 — 10:13 — page } 195 \text { — \#1 }
$$

\title{
Les mathématiques dans le grand public et dans l'enseignement : quelques éléments d'une analyse didactique
}

\author{
André Antibi et JeAn Bichara
}

Abstract. The paper looks for reaction of the public at large that is people out of educational system, concerning the mathematical exercises. We can see some results about:

- impact of terms on the motivation

- the effects of the traditional didactic on the method to resolve a problem.

Résumé. Cet article cherche la réactions du grand public c.a.d. de personnes hors système scolaire, de nombreuses annèes après avoir terminé leurs études vis à vis des exercises mathématiques.

Nous présentons quelques résultats concernant les points suivants :

- l'impact de l' « habillage » d'un énoncé sur la motivation

- les effets de l'absence d'un contrat didactique traditionnel sur la manière de résoudre un problème.

Key words and phrases: mathématique hors système scolaire, Mathematics out of school. ZDM Subject Classification: D10, D60. 


$$
\text { "antibi" — 2007/8/17 — 10:13 — page } 196 \text { — \#2 }
$$

\section{Introduction}

Usuellement, les recherches en didactique des mathématiques concernent l'enseignement en situation scolaire traditionnelle. Dans cet article, nous étudions certaines réactions du « grand public » vis à vis des mathématiques. Cette étude est favorisée par la longue expérience de Jean Bichara dans le domaine des mathématiques pour tous à la radio et à la télévision en Guadeloupe.

Nous présentons ici quelques résultats, extraits de sa thèse [2], concernant les points suivants :

- le niveau mathématique de personnes hors système scolaire, de nombreuses années après avoir terminé leurs études

- l'impact de l'« habillage » d'un énoncé sur la motivation [1]

- les effets de l'absence d'un contrat didactique traditionnel sur la manière de résoudre un problème $[3,4]$.

I. Que reste-t-il de l'enseignement des mathématiques après l'école?

Nous nous proposons dans ce paragraphe d'étudier les réactions de personnes vis à vis de certains problèmes classiques de mathématiques, plusieurs années après avoir terminé leurs études. Les résultats des enquêtes que nous avons effectuées présentent un double intérêt :

- ils permettent de mieux percevoir le niveau mathématique de personnes hors système scolaire

- ces résultats pourraient permettre également de faire évoluer l'enseignement traditionnel. En effet, nous émettons l'hypothèse que le niveau de compréhension et de mémorisation de certaines notions, plusieurs années après l'école, peut permettre de mieux percevoir les problèmes didactiques liés à l'enseignement de ces notions.

\section{Présentation du test.}

Nous avons choisi de tester les problèmes classiques suivants, qui ne font appel qu'à des connaissances de Collège : proportionnalité, résolution d'une équation du premier degré, théorème de Pythagore, parallélogramme, triangle isocèle, équation de droite, théorème de Thalès et trigonométrie.

10 exercices de mathématiques de type scolaire, du niveau Collège, présentés ci-dessous, ont été proposés à 237 personnes du « grand public », sur leur lieu de 


$$
\text { "antibi" — 2007/8/17 - 10:13 — page } 197 \text { — \#3 }
$$

travail et dans des lieux publics (Caisse d'allocation familiale, Caisse de Sécurité Sociale, Bureau des Douanes, salles d'attente de médecin, salon de coiffure, aéroport, entrée-sortie de supermarchés, Trésor Public, EDF, entreprises, Rectorat, établissements scolaires, Centre d'Apprentis, ANPE, Mairies, Commissariat de Police, salle des professeurs de Collège et de Lycée etc...)

Ces lieux ont été choisis car ce sont des endroits où les personnes attendent le plus souvent et par conséquent peuvent-être plus disposées pour répondre à un questionnaire et où elles sont assises.

\section{Précisions sur les conditions de passation.}

- Jean Bichara a effectué ces tests.

- Après s'être présenté comme professeur de mathématiques, et avoir indiqué les objectifs de notre travail, il a demandé aux personnes interrogées depuis quand elles n'avaient plus fait de mathématiques de type scolaire ou universitaire.

- Il n'y a pas eu de limite de temps imposé.

- La passation du questionnaire a duré en moyenne entre 15 et 20 minutes.

- Il n'y a eu aucune indication de sa part même après de nombreuses demandes ${ }^{1}$.

- Aucune justification n'était exigée : les personnes qui le souhaitaient avaient la possibilité d'utiliser la fiche du questionnaire pour préciser certains points : calcul, méthodes utilisées...

- Celles qui disposaient d'une calculatrice l'ont prise spontanément sans même demander si elle était permise.

- Quand le questionnaire a été rendu, celles qui le souhaitaient ont été encouragées à expliciter oralement leur démarche.

- Toutes les personnes qui ont accepté d'être interrogées ont essayé de répondre à toutes les questions.

- Beaucoup de personnes de niveau scolaire « école primaire » ont refusé de répondre au questionnaire. Ces personnes sont souvent âgées.

\section{Remarque : Les mauvais souvenirs scolaires.}

La forme scolaire du questionnaire, a semblé mettre mal à l'aise et raviver, chez certains, de mauvais souvenirs. D'après leurs réactions, un peu plus de la moitié de ces 237 personnes ont semblé penser qu'un professeur de mathématiques

${ }^{1}$ Des explications ont toujours été données à la fin du test. 


$$
\text { "antibi" — 2007/8/17 — 10:13 — page } 198 \text { — \#4 }
$$

ne pouvait leur poser un problème que pour les «piéger», les mettre en difficulté ou évaluer leur intelligence. Beaucoup de ces personnes ont semblé craindre d'être «humiliées » en ne sachant pas faire. C'est clairement exprimé dans cette réaction : "J'ai été mal à l'aise devant cette feuille parce qu'on se dit, ces petits exercices, c'est humiliant de ne pas savoir les faire ", ou dans celle-là : "C'est frustrant de ne pas savoir faire. J'ai honte de ne pas trouver. J'ai tout oublié. »

Constitution de l'échantillon par niveau d'étude et par classe d'âge.

\begin{tabular}{|c|c|c|c|c|}
\hline & $\begin{array}{c}\text { de } 20 \text { à } 30 \text { ans } \\
(73)\end{array}$ & $\begin{array}{c}\text { de } 31 \text { à } 50 \text { ans } \\
(119)\end{array}$ & $\begin{array}{c}\text { plus de } 51 \text { ans } \\
(45)\end{array}$ & $\begin{array}{c}\text { Total } \\
(237)\end{array}$ \\
\hline Primaire, & $34,25 \%$ & $22,69 \%$ & $22,22 \%$ & $26,16 \%$ \\
Collège & $(25)$ & $(27)$ & $(10)$ & $(62)$ \\
\hline Lycée & $34,25 \%$ & $42,02 \%$ & $35,55 \%$ & $38,40 \%$ \\
& $(25)$ & $(50)$ & $(16)$ & $(91)$ \\
\hline Supérieur & $15,06 \%$ & $19,32 \%$ & $26,67 \%$ & $19,41 \%$ \\
littéraire & $(11)$ & $(23)$ & $(12)$ & $(46)$ \\
\hline Supérieur & $16,44 \%$ & $15,97 \%$ & $15,56 \%$ & $16,03 \%$ \\
scientifique & $(12)$ & $(19)$ & $(7)$ & $(38)$ \\
\hline
\end{tabular}

N.B. :

- Les effectifs sont indiqués entre parenthèses.

- Dans la rubrique Lycée ont été regroupées les personnes de niveau lycée et les personnes de niveau lycée professionnel, BTS compris.

- Les personnes regroupées, respectivement dans les catégories « supérieur littéraire » et « supérieur scientifique », ont le niveau au moins « bac $+3 »$.

\section{Présentation des exercices du test.}

Présentation de l'exercice 1.

\section{Exercice 1.}

Une voiture consomme 7 litres d'essence pour parcourir 80 kilomètres.

Combien d'essence consommera t-elle pour parcourir 88 kilomètres dans les mêmes conditions?

$\square$ pour parcourir 88 kilomètres elle consommera

$\square$ je ne sais pas

$\square$ je ne m'en souviens plus 


$$
\text { "antibi" — 2007/8/17 — 10:13 — page } 199 \text { — \#5 }
$$

Dans toute la suite cet exercice sera désigné par « proportionnalité » ou « essence $»$.

La réponse attendue est : 7,7 litres

L'exercice peut être résolu en :

- remarquant que 8 est égal à un dixième de 80 , ou bien que c'est $10 \%$ de 80. La voiture consommera en plus un dixième des 7 litres d'essence, soit $7,7 \mathrm{~L}$.

- utilisant une règle de trois ou en utilisant un produit en croix

\section{Remarque.}

De tout le questionnaire, cet exercice est le seul dont l'énoncé est en relation avec la vie de tous les jours.

\section{Présentation de l'exercice 2.}

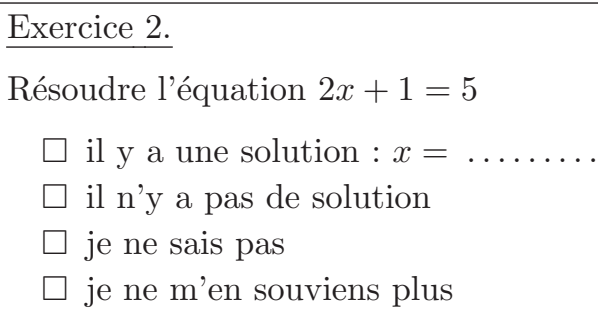

Dans toute la suite cet exercice sera désigné par « équation ».

La réponse attendue est $x=2$

L'équation peut-être résolue en remarquant que l'on part de $x$, encore faut-il savoir ce que représente $x$, on le multiplie par 2 et on ajoute 1 au résultat. Pour avoir $x$ on fait le retour inverse : soustraire 1 et diviser par 2 .

On peut remarquer que si on remplace $x$ par 2 cela marche.

On peut aussi écrire

$$
\begin{aligned}
2 x+1 & =5 \\
2 x & =5-1 \\
2 x & =4 \\
x & =2
\end{aligned}
$$




$$
\text { "antibi" — 2007/8/17 — 10:13 — page } 200-\# 6
$$

Présentation de l'exercice 3.

\section{Exercice 3.}

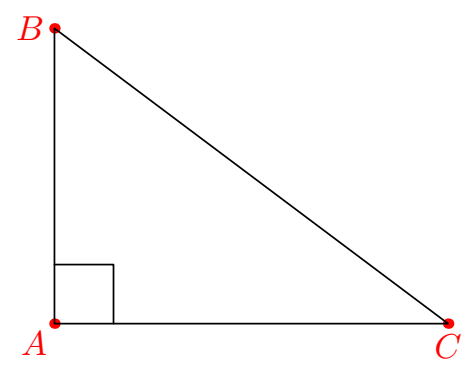

$A B C$ est un triangle rectangle en $A$. Le segment $[A B]$ mesure 3 centimètres, le segment $[A C]$ mesure 4 centimètres. Combien mesure le segment $[B C]$ ? (sur la figure les mesures ne sont pas respectées)

$\square$ le segment $[B C]$ mesure $\ldots \ldots \ldots$.

$\square$ je ne sais pas

$\square$ je ne m'en souviens plus

Dans toute la suite, cet exercice sera désigné par « Pythagore »

La réponse attendue est : le segment $[B C]$ mesure $5 \mathrm{~cm}$.

Il suffit d'utiliser le théorème de Pythagore.

On peut aussi utiliser le fait que $(3,4,5)$ est le premier triplet pythagoricien, mais, bien sûr, nous n'attendions pas ce type de réponse.

Présentation de l'exercice 3 bis.

Exercice 3 bis.

Quel est le théorème qui permet de calculer la mesure du segment $[B C]$ ?

$\square$ je sais, c'est le théorème

$\square$ je ne sais pas

$\square$ je ne m'en souviens plus

Dans toute la suite cet exercice sera désigné par « Pythagore bis »

La réponse attendue est : le théorème de Pythagore. 


$$
\text { "antibi" — 2007/8/17 — 10:13 — page } 201 \text { — \#7 }
$$

Présentation de l'exercice 4.

Exercice 4.

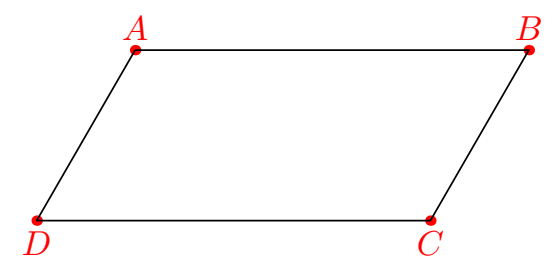

La droite $(A B)$ est parallèle à la droite $(D C)$. La droite $(A D)$ est parallèle à la droite $(B C)$.

Cette figure est-elle?

$\square$ un carré

$\square$ un rectangle

$\square$ un losange

$\square$ un parallélogramme

$\square$ je ne sais pas

$\square$ je ne m'en souviens plus

Dans toute la suite cet exercice sera désigné par «parallélogramme » La réponse attendue est : «parallélogramme ».

Présentation de l'exercice 5.

\section{Exercice 5.}

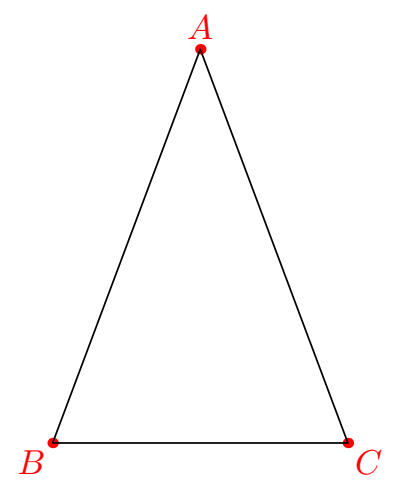




$$
\text { "antibi" — 2007/8/17 — 10:13 — page } 202 \text { — \#8 }
$$

Dans le triangle $A B C$ les côtés $A B$ et $A C$ ont la même longueur. $(A B=A C)$ Quelle est la nature du triangle $A B C$ ?

$\square$ le triangle $A B C$ est un triangle ........

$\square$ je ne sais pas

$\square$ je ne m'en souviens plus

Dans toute la suite cet exercice sera désigné par « isocèle ».

La réponse attendue est : « Le triangle $A B C$ est un triangle isocèle »

Présentation de l'exercice 5 bis.

Exercice 5 bis.

L'angle $\hat{B}$ est-il égal à l'angle $\hat{C}$ ?

$\square$ oui

$\square$ non

$\square$ je ne sais pas

$\square$ je ne m'en souviens plus

Dans toute la suite cet exercice sera désigné par « angle »

La réponse attendue est : « oui ».

Présentation de l'exercice 6.

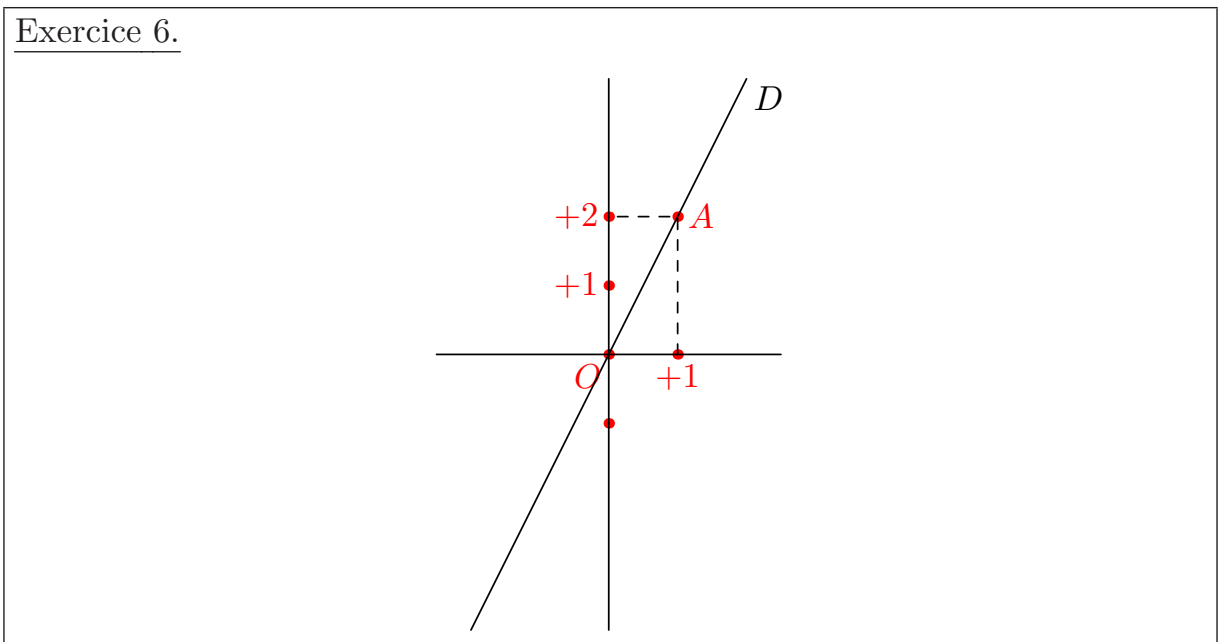

$\bigoplus$ 


$$
\text { "antibi" — 2007/8/17 — 10:13 — page } 203 \text { — \#9 }
$$

Quelle est l'équation de la droite $(D)$ passant par $O$ et $A$ ?

$\square$ je sais c'est $\ldots . . \ldots$.

$\square$ je ne sais pas

$\square$ je ne m'en souviens plus

Dans toute la suite cet exercice sera désigné par : « équation de droite »

La réponse attendue est : $y=2 x$

\section{Présentation de l'exercice \%.}

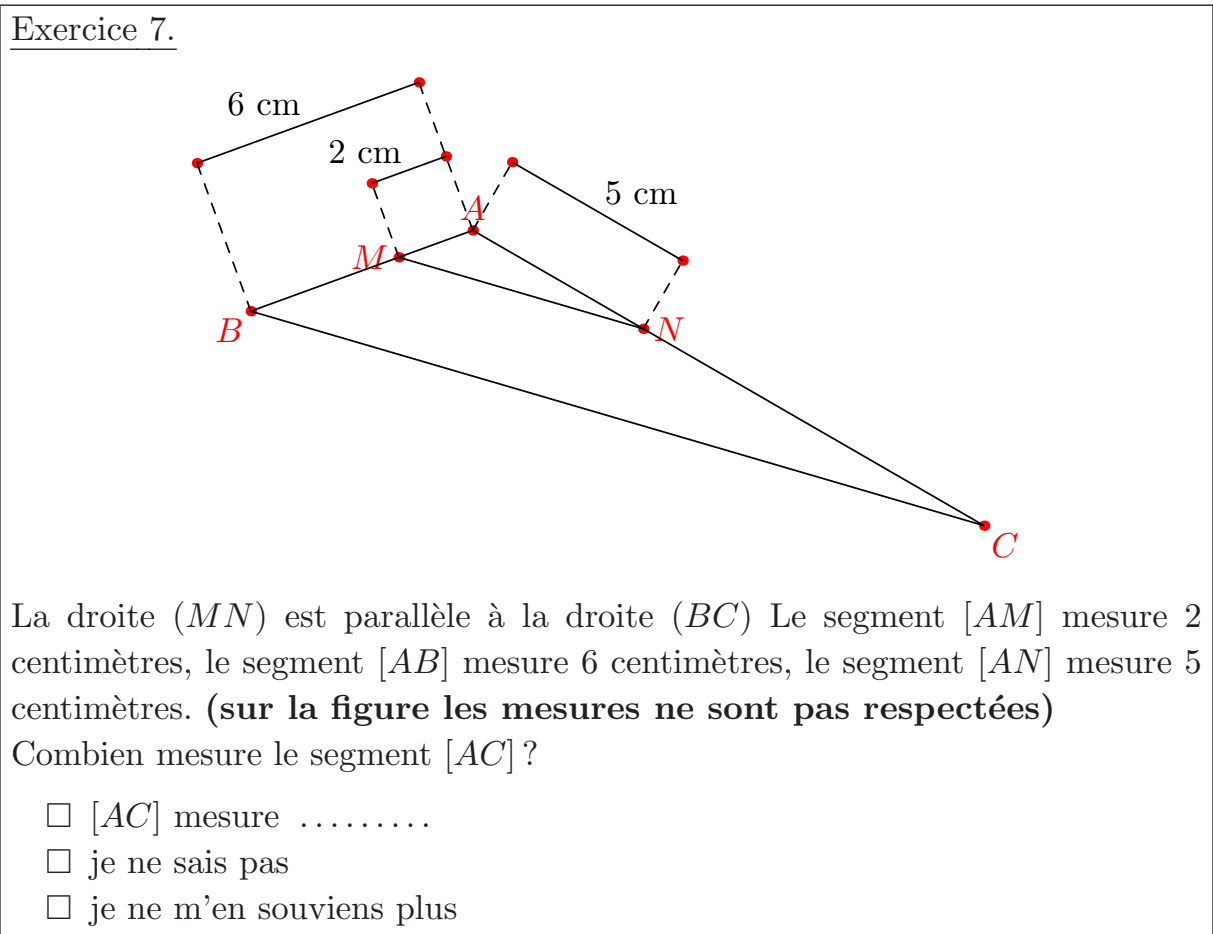

Dans toute la suite cet exercice sera désigné par « Thalès »

La réponse attendue est $A C=15 \mathrm{~cm}$. Il y a différentes possibilités pour l'obtenir :

(1) Première possibilité : (Thalès)

$$
\text { de } \frac{A N}{A C}=\frac{A M}{A B} \text { on tire : } A C=A N \times \frac{A B}{A M}=5 \times \frac{6}{2}=15
$$


(2) Deuxième possibilité :

$$
\text { Comme } A B=3 \times A M \text { alors } A C=3 \times A N=3 \times 5=15
$$

Présentation de l'exercice 7 bis.

Exercice 7 bis.

Quel est le théorème qui permet de calculer la mesure du segment $[A C]$ ?

$\square$ je sais, c'est le théorème

$\square$ je ne sais pas

$\square$ je ne m'en souviens plus

Dans toute la suite cet exercice sera désigné par : « Thalès bis »

La réponse attendue est : «le théorème de Thalès ».

Présentation de l'exercice 8.

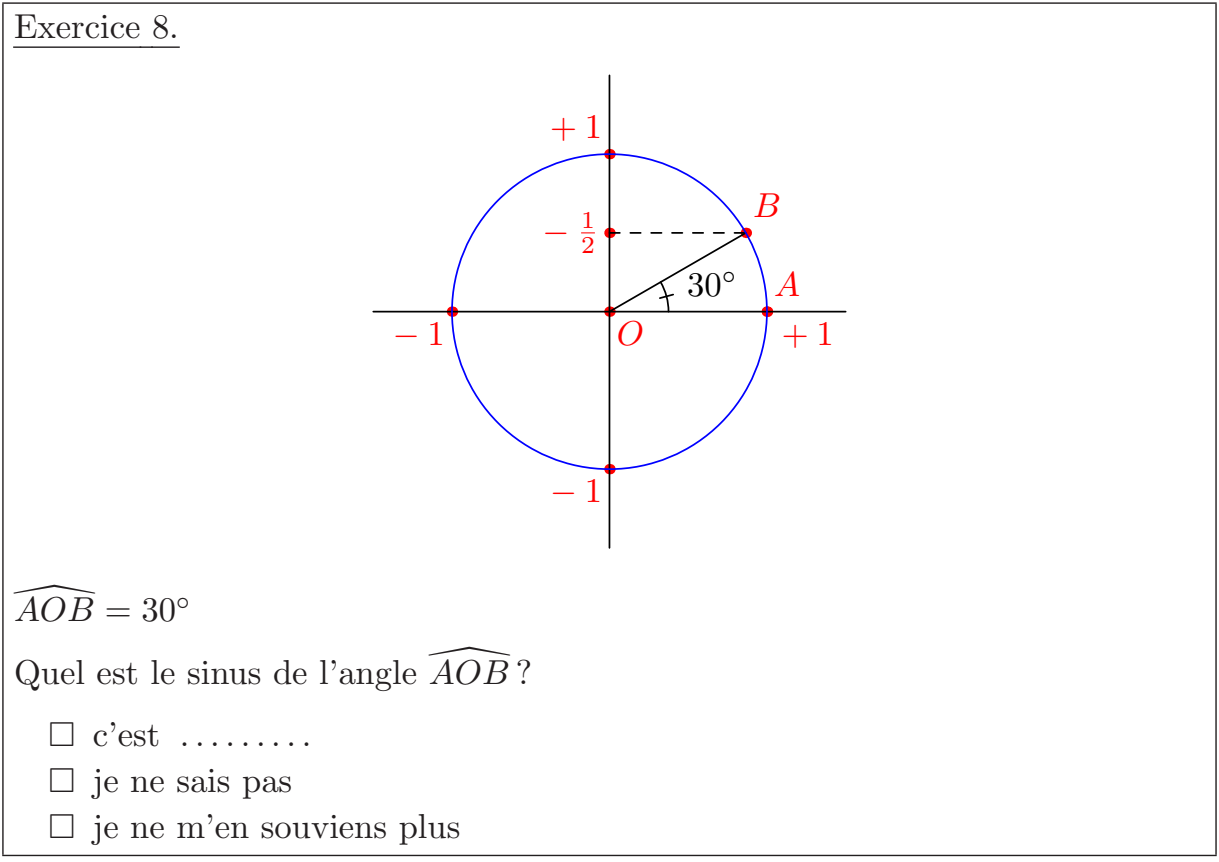

Dans toute la suite cet exercice sera désigné par « sinus » ou « trigonométrie » La réponse attendue est $: \sin 30^{\circ}=\frac{1}{2}=0,5$ 


$$
\text { "antibi" — 2007/8/17 — 10:13 — page 205 — \#11 }
$$

Résultats

\begin{tabular}{|c|c|c|c|c|c|}
\hline \multicolumn{6}{|c|}{ Réussite aux exercices en fonction du niveau d'étude } \\
\hline Niveau d'étude & $\begin{array}{c}\text { Primaire } \\
\text { Collège } \\
(62)\end{array}$ & $\begin{array}{c}\text { Lycée } \\
(92)\end{array}$ & $\begin{array}{c}\text { Supérieur } \\
\text { littéraire } \\
(47)\end{array}$ & $\begin{array}{c}\text { Supérieur } \\
\text { scientifique } \\
(36)\end{array}$ & $\begin{array}{l}\text { Total } \\
(237)\end{array}$ \\
\hline Essence & $\begin{array}{c}22,58 \% \\
(14)\end{array}$ & $\begin{array}{c}57,61 \% \\
(53)\end{array}$ & $\begin{array}{c}59,57 \% \\
(28)\end{array}$ & $\begin{array}{c}100 \% \\
(36)\end{array}$ & $\begin{array}{c}55,27 \% \\
(131)\end{array}$ \\
\hline Equation & $\begin{array}{c}30,65 \% \\
(19)\end{array}$ & $\begin{array}{c}65,22 \% \\
(60)\end{array}$ & $\begin{array}{c}72,34 \% \\
(34)\end{array}$ & $\begin{array}{c}100 \% \\
(36)\end{array}$ & $\begin{array}{c}62,87 \% \\
(149)\end{array}$ \\
\hline Pythagore & $\begin{array}{c}17,74 \% \\
(11)\end{array}$ & $\begin{array}{c}38,04 \% \\
(35)\end{array}$ & $\begin{array}{c}34,04 \% \\
(16)\end{array}$ & $\begin{array}{c}97,22 \% \\
(35)\end{array}$ & $\begin{array}{c}40,93 \% \\
\quad(97)\end{array}$ \\
\hline Pythagore bis & $\begin{array}{c}22,58 \% \\
(14)\end{array}$ & $\begin{array}{c}45,65 \% \\
(42)\end{array}$ & $\begin{array}{c}51,06 \% \\
(24)\end{array}$ & $\begin{array}{c}91,67 \% \\
(33)\end{array}$ & $\begin{array}{c}47,68 \% \\
(113)\end{array}$ \\
\hline Parallélogramme & $\begin{array}{c}50 \% \\
(31)\end{array}$ & $\begin{array}{c}71,74 \% \\
(66)\end{array}$ & $\begin{array}{c}87,23 \% \\
(41)\end{array}$ & $\begin{array}{c}97,22 \% \\
(35)\end{array}$ & $\begin{array}{l}73 \% \\
(173)\end{array}$ \\
\hline Isocèle & $\begin{array}{c}41,94 \% \\
(26)\end{array}$ & $\begin{array}{c}60,87 \% \\
(56)\end{array}$ & $\begin{array}{c}78,72 \% \\
(37)\end{array}$ & $\begin{array}{c}94,44 \% \\
(34)\end{array}$ & $\begin{array}{c}64,56 \% \\
(153)\end{array}$ \\
\hline Isocèle bis & $\begin{array}{c}70,97 \% \\
(44)\end{array}$ & $\begin{array}{c}78,26 \% \\
(72)\end{array}$ & $\begin{array}{c}95,74 \% \\
(45)\end{array}$ & $\begin{array}{c}94,44 \% \\
(34)\end{array}$ & $\begin{array}{c}82,28 \% \\
(195)\end{array}$ \\
\hline Equation de droite & $\begin{array}{l}0 \% \\
(0)\end{array}$ & $\begin{array}{c}8,70 \% \\
(8)\end{array}$ & $\begin{array}{c}2,13 \% \\
(1)\end{array}$ & $\begin{array}{c}72,22 \% \\
(26)\end{array}$ & $\begin{array}{c}14,77 \% \\
(35)\end{array}$ \\
\hline Thalès & $\begin{array}{c}4,84 \% \\
(3)\end{array}$ & $\begin{array}{c}20,65 \% \\
(19)\end{array}$ & $\begin{array}{c}12,77 \% \\
(6)\end{array}$ & $\begin{array}{c}75 \% \\
(27)\end{array}$ & $\begin{array}{c}23,21 \% \\
(55)\end{array}$ \\
\hline Thalès bis & $\begin{array}{c}4,84 \% \\
(3)\end{array}$ & $\begin{array}{c}21,74 \% \\
(20)\end{array}$ & $\begin{array}{c}25,53 \% \\
(12)\end{array}$ & $\begin{array}{c}72,22 \% \\
(26)\end{array}$ & $\begin{array}{c}25,74 \% \\
(61)\end{array}$ \\
\hline Sinus $30^{\circ}$ & $\begin{array}{c}1,61 \% \\
(1)\end{array}$ & $\begin{array}{c}6,52 \% \\
(6)\end{array}$ & $\begin{array}{c}4,26 \% \\
(2)\end{array}$ & $\begin{array}{c}36,11 \% \\
(13)\end{array}$ & $\begin{array}{c}9,28 \% \\
(22)\end{array}$ \\
\hline
\end{tabular}

N.B. : Le nombre entre parenthèses dans chaque case est le nombre de réponses.

\section{Commentaires :}

- Dans l'ensemble des trois catégories « Primaire », « Lycée » et « Supérieur scientifique », les pourcentages de réussite sont proportionnels au niveau d'étude. Ceci était prévisible, a priori. Les différences entre le pourcentage de réussite sont nettes.

- Entre les catégories « Lycée » et « Supérieur littéraire », le sens de variation des pourcentages diffère selon les questions. Ceci peut être expliqué 


$$
\text { "antibi" — 2007/8/17 — 10:13 — page } 206 \text { — \#12 }
$$

par le fait que des personnes de niveau «lycée » ont pu effectuer leurs études dans des sections scientifiques, contrairement à certaines personnes de niveau «Supérieur littéraire ». Notons que, pour plusieurs exercices, les pourcentages de réussite dans ces deux catégories sont de même ordre.

- Dans chacune des trois catégories "Primaire ", "Lycée " et «Supérieur littéraire », classons les pourcentages de réussite par ordre décroissant; les trois classements ainsi obtenus sont pratiquement identiques. Ainsi le taux de réussite dépend du niveau d'étude, mais la difficulté relative des points traités ne semble pas en dépendre.

- Dans la catégorie «Supérieur scientifique », les résultats sont meilleurs, certes, mais d'autre part, on ne retrouve pas le classement précédent concernant les pourcentages de réussite, sauf pour les exercices « équation de droite », et surtout « sinus » qui, ici aussi, sont en fin de classement.

- Le mauvais score obtenu à l'exercice « sinus» dans toutes les catégories peut être expliqué par le fait que l'aspect purement conventionnel d'une définition n'aide pas à mémoriser. D'ailleurs, de nombreux élèves, en situation scolaire, commettent des erreurs de ce type en trigonométrie.

- Exercice « essence » : une surprise concernant la proportionnalité? C'est certainement le point où les résultats sont les plus surprenants, et même inquiétants. En dehors de la catégorie «Supérieur scientifique » où tous les résultats sont corrects, le taux de réussite à cet exercice est faible compte tenu de sa « simplicité ». Lors d'une analyse a priori, nous nous attendions à de meilleurs résultats sur les problèmes liés à l'enseignement de la proportionnalité : règle de trois, produits en croix, lien avec la linéarité, ... Le système scolaire met l'accent sur ce point, utile dans la vie de tous les jours. Dans la catégorie « Primaire Collège », moins d'une personne sur quatre donne la réponse; à peine un peu plus d'une sur deux dans les catégories «Lycée » et «Supérieur littéraire », pour un exercice qui ne nécessite aucun outil sophistiqué, mais seulement un peu de «bon sens » (la « méthode du dixième » suffit).

Comment doit-on interpréter une telle situation? Est-elle révélatrice d'un certain type d'échec de notre système éducatif? Enseigne-t-on trop de propriétés et insiste-t-on trop peu sur un minimum de connaissances que tout citoyen devrait posséder?

Tous ces points semblent essentiels et montrent l'intérêt d'une étude didactique sur l'enseignement des mathématiques, hors système scolaire. 


$$
\text { "antibi" — 2007/8/17 — 10:13 — page } 207 \text { — \#13 }
$$

- Les résultats obtenus à l'exercice « Thalès » sont faibles. Ici aussi il s'agit de proportionnalité, mais la situation proposée n'est pas liée à la vie quotidienne.

- Contrairement à ce que nous avions prévu dans notre analyse a priori, les résultats à l'exercice « équation» sont meilleurs qu'à l'exercice « essence ». Certes, l'équation proposée est simple, mais, pour répondre à la question, il était nécessaire de comprendre ce que signifie « résoudre une équation ».

- Signalons enfin « les bons » résultats obtenus aux exercices «parallélogramme » et « isocèle »; les définitions liées à des figures géométriques semblent être bien mémorisées dans l'ensemble.

\section{Influence de l'habillage des exercices sur la motivation}

Il est souvent question de motivation dans les débats sur l'enseignement, et, souvent, certains pensent qu'un « habillage » d'un énoncé peut le rendre plus motivant. André Antibi [1] a mis en évidence le phénomène de décalage motivationnel : les enseignants ont tendance à penser que leur motivation vis à vis d'une notion ou d'un exercice est partagée par les élèves, alors qu'en réalité, existe un décalage entre la motivation du professeur et celle de ses élèves.

Dans ce paragraphe, nous nous proposons de tester les réactions du « grand public » dans le domaine de la «motivation ».

\section{Le questionnaire}

Les trois énoncés correspondent au même problème de mathématiques. On vous demande de les lire, mais on ne vous demande pas de le résoudre.

\section{Énoncé 1}

Trouvez deux nombres dont la somme est 100 et tel que l'un d'eux est supérieur à l'autre de 50 .

\section{Énoncé 2 :}

Céline et Charlotte possèdent 100 francs à elles deux. Céline a 50 francs de plus que Charlotte. Combien possèdent chacune d'elles? 


$$
\text { "antibi" — 2007/8/17 — 10:13 — page } 208 \text { — \#14 }
$$

\section{Énoncé 3}

Pour avoir la bosse des maths, Monsieur Têtevide a bu 100 centilitres d'une potion magique composée d'extrait de racine carrée et de lait de cabri géomètre. La quantité d'extrait de racine dépassant celle de lait de 50 centilitres, quelle est la quantité de chaque ingrédient de cette potion?

Quel énoncé préférez-vous? (cocher une case)

\[ \text { aucun } \square \text { énoncé } 1 \quad \square \text { énoncé } 2 \quad \square \text { énoncé } 3 \]
Pourquoi?

Il s'agit de trois énoncés différents du même problème de mathématiques :

- un énoncé abstrait, (le premier),

- un énoncé à enrobage familier, (le second),

- un énoncé à enrobage humoristique, (le troisième).

Ils ont été proposés à différents publics :

- 157 personnes du grand public

- 631 scolaires (du CM 2 à la terminale)

- 53 enseignants de mathématiques (Primaire, Collège, Lycée)

\section{Remarques :}

La consigne, qui était de lire les trois énoncés sans les résoudre, a été rappelée oralement.

Le fait de ne pas demander la résolution a souvent été très bien accueilli que ce soit par le grand public, les élèves ou même les enseignants. Ceci a permis à des personnes du grand public de répondre aux questions, alors qu'au premier abord elles refusaient de le faire de crainte, semble t-il, d'être « humiliées ».

Beaucoup de personnes n'ont pas compris que c'était le même problème de mathématiques. Effectivement, le lien entre les énoncés 2 et 3 et l'énoncé 1 n'est pas immédiat.

\section{Catégorie « grand public».}

Le questionnaire a été proposé à 157 personnes du grand public, sur leur lieu de travail et dans des lieux publics : Caisse d'allocation familiale, Caisse de Sécurité Sociale, Trésor Public, EDF, Bureau des Douanes, Entreprises, Rectorat, Aéroport, Entrée-sortie de supermarchés, Etablissements scolaires, Centre d'Apprentis, ANPE, Mairies, Commissariat de Police, salle d'attente de médecin, salon 
de coiffure. Dans cette catégorie « grand public» ont été exclus les enseignants de mathématiques et les étudiants en mathématiques.

L'échantillon est constitué de personnes qui ont bien voulu accepter de répondre au questionnaire.

Les personnes de faible niveau scolaire ont souvent refusé de répondre.

\section{Constitution de l'échantillon.}

Il est constitué de 157 personnes réparties par niveau d'étude :

- 23 du niveau Ecole primaire

- 27 du niveau Collège

- 36 du niveau Lycée

- 39 du niveau supérieur littéraire

- 32 du niveau supérieur scientifique (non matheux).

\section{Remarque.}

Le nombre de personnes de niveau d'études « supérieur» est plus élevé car ces personnes acceptent de répondre plus facilement à une enquête portant sur les mathématiques que les autres.

\section{Résultats.}

Tableau : Enoncé préféré des personnes du grand public.

\begin{tabular}{|c|c|c|c|c|c|c|}
\hline & $\begin{array}{c}\text { Primaire } \\
(23)\end{array}$ & $\begin{array}{c}\text { Collège } \\
(27)\end{array}$ & $\begin{array}{c}\text { Lycée } \\
(36)\end{array}$ & $\begin{array}{l}\text { Sup } \\
\text { Litt } \\
(39)\end{array}$ & $\begin{array}{c}\text { Sup } \\
\text { Science } \\
(32)\end{array}$ & $\begin{array}{l}\text { Total } \\
(157)\end{array}$ \\
\hline Aucun & $\begin{array}{c}4,34 \% \\
(1)\end{array}$ & $\begin{array}{c}11,11 \% \\
(3)\end{array}$ & $\begin{array}{c}8,34 \% \\
(3)\end{array}$ & $\begin{array}{c}5,13 \% \\
(2)\end{array}$ & $\begin{array}{c}3,13 \% \\
(1)\end{array}$ & $\begin{array}{c}6,37 \% \\
(10)\end{array}$ \\
\hline $\begin{array}{l}\text { Enoncé } 1 \\
\text { (abstrait) }\end{array}$ & $\begin{array}{c}17,40 \% \\
(4)\end{array}$ & $\begin{array}{c}11,11 \% \\
(3)\end{array}$ & $\begin{array}{c}27,77 \% \\
(10)\end{array}$ & $\begin{array}{c}20,51 \% \\
(8)\end{array}$ & $\begin{array}{c}53,12 \% \\
(17)\end{array}$ & $\begin{array}{c}26,75 \% \\
(42)\end{array}$ \\
\hline $\begin{array}{l}\text { Enoncé } 2 \\
\text { (familier) }\end{array}$ & $\begin{array}{c}65,22 \% \\
(15)\end{array}$ & $\begin{array}{c}55,56 \% \\
(15)\end{array}$ & $\begin{array}{c}50 \% \\
(18)\end{array}$ & $\begin{array}{c}48,72 \% \\
(19)\end{array}$ & $\begin{array}{c}25 \% \\
(8)\end{array}$ & $\begin{array}{c}47,77 \% \\
(75)\end{array}$ \\
\hline $\begin{array}{l}\text { Enoncé } 3 \\
\text { (humour) }\end{array}$ & $\begin{array}{c}8,70 \% \\
(2)\end{array}$ & $\begin{array}{c}22,22 \% \\
(6)\end{array}$ & $\begin{array}{c}11,11 \% \\
(4)\end{array}$ & $\begin{array}{c}25,64 \% \\
(10)\end{array}$ & $\begin{array}{c}18,75 \% \\
(6)\end{array}$ & $\begin{array}{c}17,83 \% \\
(28)\end{array}$ \\
\hline Autre & $\begin{array}{c}4,34 \% \\
(1)\end{array}$ & $\begin{array}{l}0 \% \\
(0)\end{array}$ & $\begin{array}{c}2,78 \% \\
(1)\end{array}$ & $\begin{array}{l}0 \% \\
(0)\end{array}$ & $\begin{array}{c}0 \% \\
(0)\end{array}$ & $\begin{array}{c}1,27 \% \\
(2)\end{array}$ \\
\hline
\end{tabular}

N.B. : Nous avons regroupé dans la rubrique lycée les personnes de niveau lycée professionnel et celle de niveau lycée général. 


$$
\text { "antibi" — 2007/8/17 — 10:13 — page } 210 \text { — \#16 }
$$

\section{Commentaires}

- L'énoncé préféré est l'énoncé 2 ; cette préférence est plus nette dans la catégorie « primaire».

- L'énoncé 3 est peu apprécié, ce qui peut surprendre dans la mesure où l'enquête est effectuée hors cadre scolaire ${ }^{2}$. On peut interpréter ceci en termes de contrat didactique; mais ici il s'agit d'un contrat d'un type très particulier, hors système scolaire. Plus précisément, lorsque l'on présente des énoncés de mathématiques hors système scolaire, (donc hors évaluation), en demandant de ne pas les résoudre, les réflexes de type scolaire sont toujours présents : il ne s'agit pas de «rigoler» mais de faire des maths, et donc de choisir l'exercice qui se prête le mieux à une résolution éventuelle, même si elle n'est pas demandée.

- Cette interprétation est tout à fait confirmée par les raisons du choix des personnes interrogées : c'est l'argument de facilité qui apparaît nettement le plus souvent.

\section{Catégorie élèves.}

Voici la répartition des élèves qui ont répondu à ce questionnaire :

- 144 élèves de 10 ans

- 76 élèves de 11 ans

- 62 élèves de 12 ans

- 63 élèves de 13 ans

- 45 élèves de 14 ans

- 68 élèves de 15 ans, enseignement professionel

- 38 élèves de 16 ans, enseignement professionel

- 21 élèves de 17 ans, enseignement professionel

- 63 élèves de 15 ans, enseignement général

- 32 élèves de 16 ans, série scientifique

- 19 élèves de 17 ans, série scientifique.

\footnotetext{
${ }^{2}$ Jean Bichara présentateur d'exercices de mathématiques à la radio et à la télévision a été surpris par ces résultats : il pensait que l'énoncé 3 connaîtrait un succès bien plus important dans le « grand public».
} 


$$
\text { "antibi" — 2007/8/17 — 10:13 — page } 211 \text { — \#17 }
$$

Ces questionnaires ont été proposés en présence de l'enseignant ${ }^{3}$ de la classe, durant une demi-heure environ.

\section{Résultats}

\begin{tabular}{|c|c|c|c|c|c|}
\hline \multicolumn{7}{|c|}{ Enoncé préféré des élèves Primaire, Collège } \\
\hline & $\begin{array}{c}10 \text { ans } \\
(144)\end{array}$ & $\begin{array}{c}11 \text { ans } \\
(76)\end{array}$ & $\begin{array}{c}12 \text { ans } \\
(62)\end{array}$ & $\begin{array}{c}13 \text { ans } \\
(63)\end{array}$ & $\begin{array}{c}14 \text { ans } \\
(45)\end{array}$ \\
\hline Aucun & $4,17 \%$ & $3,95 \%$ & $6,45 \%$ & $6,35 \%$ & $6,66 \%$ \\
& $(6)$ & $(3)$ & $(4)$ & $(4)$ & $(3)$ \\
\hline Enoncé 1 & $14,58 \%$ & $14,47 \%$ & $17,74 \%$ & $17,46 \%$ & $17,79 \%$ \\
(abstrait) & $(21)$ & $(11)$ & $(11)$ & $(11)$ & $(8)$ \\
\hline Enoncé 2 & $61,11 \%$ & $59,21 \%$ & $59,68 \%$ & $71,43 \%$ & $73,33 \%$ \\
(familier) & $(88)$ & $(45)$ & $(37)$ & $(45)$ & $(33)$ \\
\hline Enoncé 3 & $18,75 \%$ & $19,74 \%$ & $16,13 \%$ & $4,76 \%$ & $2,22 \%$ \\
(Humour) & $(27)$ & $(15)$ & $(10)$ & $(3)$ & $(1)$ \\
\hline Autre & $1,39 \%$ & $2,64 \%$ & $0 \%$ & $0 \%$ & $0 \%$ \\
& $(2)$ & $(2)$ & $(0)$ & $(0)$ & $(0)$ \\
\hline
\end{tabular}

Enoncé préféré des élèves de Lycée

\begin{tabular}{|c|c|c|c|c|c|c|}
\hline & $\begin{array}{c}15 \text { ans } \\
\text { PRO }^{*} \\
(68)\end{array}$ & $\begin{array}{c}17 \text { ans } \\
\text { PRO }^{*} \\
(21)\end{array}$ & $\begin{array}{c}16 \text { ans } \\
\text { PRO }^{*} \\
(38)\end{array}$ & $\begin{array}{c}15 \text { ans } \\
(63)\end{array}$ & $\begin{array}{c}16 \text { ans, } \\
\text { Scientif. } \\
(32)\end{array}$ & $\begin{array}{c}17 \text { ans, } \\
\text { Scientif. } \\
(19)\end{array}$ \\
\hline Aucun & $\begin{array}{c}8,83 \% \\
(6)\end{array}$ & $\begin{array}{c}4,76 \% \\
(1)\end{array}$ & $\begin{array}{c}5,26 \% \\
(2)\end{array}$ & $\begin{array}{c}3,17 \% \\
(2)\end{array}$ & $\begin{array}{c}0 \% \\
(0)\end{array}$ & $\begin{array}{c}0 \% \\
(0)\end{array}$ \\
\hline Enoncé 1 & $10,29 \%$ & $23,81 \%$ & $26,32 \%$ & $22,19 \%$ & $25 \%$ & $21,05 \%$ \\
(Abstrait) & $(7)$ & $(5)$ & $(10)$ & $(14)$ & $(8)$ & $(4)$ \\
\hline Enoncé 2 & $73,53 \%$ & $61,91 \%$ & $65,79 \%$ & $66,57 \%$ & $65,63 \%$ & $63,16 \%$ \\
(familier) & $(50)$ & $(13)$ & $(25)$ & $(42)$ & $(21)$ & $(12)$ \\
\hline Enoncé 3 & $7,35 \%$ & $9,52 \%$ & $2,63 \%$ & $8,07 \%$ & $9.37 \%$ & $15,79 \%$ \\
(Humour) & $(5)$ & $(2)$ & $(1)$ & $(5)$ & $(3)$ & $(3)$ \\
\hline Autre & $0 \%$ & $0 \%$ & $0 \%$ & $0 \%$ & $0 \%$ & $0 \%$ \\
& $(0)$ & $(0)$ & $(0)$ & $(0)$ & $(0)$ & $(0)$ \\
\hline
\end{tabular}

${ }^{3}$ Nous remercions les enseignants qui ont permis le passage des questionnaires dans leur classe : Simone Pasbeau, Gisèle Dufresne, Marlène Attam-Kassigadou, Sidonie Bourguignon, Frédéric Louvet, Marne, Myriam Thorin, Marie Lisette, Philippe Delanay, Michel Zénon, Daniel Chataigne, Louis Ogier, Alain Magen, Aude Séverin, Alex Bissainte, Nestar. 
N.B. : Rappelons que les qualificatifs « abstrait », « vie quotidienne » et « humour $»$ ne figuraient pas dans le questionnaire.

\section{Commentaire.}

Le comportement des élèves est analogue à celui du grand public. Ici aussi, c'est l'énoncé 2 qui est préféré, et c'est essentiellement l'argument « facilité » qui justifie ce choix.

\section{Catégorie Enseignants.}

53 enseignants ont été interrogés.

Tous enseignent depuis plus de 5 ans.

L'échantillon est constitué de :

- 14 enseignants du Primaire,

- 24 enseignants de Collège

- 15 enseignants de Lycée.

\section{Remarque :}

La question : «Quel énoncé préférez-vous?» a gêné les enseignants. Presque tous, pensent : «quel énoncé préférez-vous pour les élèves?». Il semble que lorsque l'on demande à un enseignant «quel énoncé préférez-vous», il pense inconsciemment, « quel énoncé préférez-vous pour vos élèves?».

\section{Les résultats :}

\begin{tabular}{|c|c|c|c|c|}
\hline \multicolumn{5}{|c|}{ Enoncé motivant d'après les enseignants } \\
\hline & $\begin{array}{c}\text { Primaire } \\
(14)\end{array}$ & $\begin{array}{c}\text { Collège } \\
(24)\end{array}$ & $\begin{array}{c}\text { Lycée } \\
(15)\end{array}$ & $\begin{array}{c}\text { Total } \\
(53)\end{array}$ \\
\hline \multirow{2}{*}{ Aucun } & $0 \%$ & $4,17 \%$ & $0 \%$ & $1,89 \%$ \\
& $(0)$ & $(1)$ & $(0)$ & $(1)$ \\
\hline Enoncé 1 & $7,14 \%$ & $12,50 \%$ & $6,67 \%$ & $9,43 \%$ \\
(abstrait) & $(1)$ & $(3)$ & $(1)$ & $(5)$ \\
\hline Enoncé 2 & $78,57 \%$ & $75,00 \%$ & $80 \%$ & $77,35 \%$ \\
(familier) & $(11)$ & $(18)$ & $(12)$ & $(41)$ \\
\hline Enoncé 3 & $17,40 \%$ & $8,33 \%$ & $13,33 \%$ & $11,32 \%$ \\
(Humour) & $(2)$ & $(2)$ & $(2)$ & $(6)$ \\
\hline Autre & $0 \%$ & $0 \%$ & $0 \%$ & $0 \%$ \\
& $(0)$ & $(0)$ & $(0)$ & $(0)$ \\
\hline
\end{tabular}




$$
\text { "antibi" — 2007/8/17 — 10:13 — page 213 — \#19 }
$$

\section{Commentaires :}

En ce qui concerne la préférence, le comportement est analogue à celui des deux catégories précédentes : l'énoncé 2 arrive nettement en tête. Mais l'analyse des justifications fournies par les enseignants pour justifier leur choix fait apparaître un net décalage avec les deux autres catégories : ici l'argument avancé est presque toujours du type «cet énoncé est plus concret 》 ou « cet énoncé est lié à la vie quotidienne ». En termes de motivation, les arguments des enseignants ne sont pas ceux des élèves ou du grand public.

\section{Effets de l'absence d'un contrat didactique traditionnel.}

Il est généralement admis que le fait de connaître un grand nombre de théorèmes permet de résoudre plus facilement des problèmes. Parfois, ce n'est pas le cas : des personnes possédant peu de connaissances scolaires peuvent résoudre plus rapidement certains exercices que des spécialistes des mathématiques. Cette situation peut être expliquée de la façon suivante :

En présence d'un problème à résoudre d'un type non usuel, on essaie de le rattacher à des problèmes analogues, et d'utiliser des outils permettant de le résoudre. Lorsque la « boîte à outils » contient beaucoup d'outils, on peut se lancer dans une solution bien trop élaborée pour le problème considéré, parfois même ne pas arriver au but. Inversement, lorsque la « boîte à outils » est presque vide, il peut arriver que des outils élémentaires qui s'y trouvent soient suffisants pour le problème considéré.

Ce phénomène se produit surtout lorsque la personne chargée de le résoudre n'a aucune idée a priori des notions rattachées à ce problème (ce n'est pas le cas à un contrôle scolaire portant sur un programme scolaire bien défini; dans ce cas on se doute que les « outils » figurant au programme doivent être utilisés).

On peut interpréter ce phénomène en terme de «contrat didactique ». Lorsque le contrat didactique n'est pas précis, une « boîte à outils » trop remplie peut ètre un handicap.

Contrairement à la situation scolaire traditionnelle où les élèves ont le même type de connaissances, le domaine du grand public se prête particulièrement bien à la mise en évidence de ce genre de phénomène. En effet, dans ce cas, les connaissances des personnes devant résoudre un problème peuvent être très différentes. 
Une première expérimentation.

Le test suivant a été proposé par André Antibi à un groupe de 40 enseignants de toutes disciplines.

Quel est le plus petit nombre entier qui s'écrit, en notation décimale, uniquement avec des chiffres 1 et qui est divisible par 31 ?

Une enseignante de français, se considérant très faible en mathématique, proposa rapidement une réponse. Elle croyait sa réponse fausse car elle était la seule à avoir « trouvée quelque chose ». Elle avait simplement posé la division, tandis que les enseignants de mathématiques, avaient cherché à «mettre en équation » et n'y étaient pas parvenus. Etant celle qui avait la « boîte à outils » la moins « chargée », elle y avait puisé le seul outil qui s'y trouvait. Tandis que d'autres, surtout parmi les enseignants de mathématiques, ont cherché à utiliser des outils bien trop sophistiqués pour le problème proposé. Vraisemblablement, s'ils avaient rencontré ce problème dans le livre de sixième ils auraient posé la division mais, dans la situation de stage, ils ne pouvaient penser que le formateur ne demande que cela. Le fait d'avoir un public hétérogène, avec des « boîtes à outils » différentes, a permis de voir apparaître ce phénomène.

Par la suite, d'autres expérimentations, du même type, ont été proposées à des enseignants par Jean Bichara [2]. Les résultats obtenus sont tout à fait analogues à ceux de l'expérimentation réalisée par André Antibi.

D'autres expérimentations dans le cadre du « grand public ».

Ces expérimentations ont été menées par Jean Bichara, et figurent dans sa thèse ${ }^{\circ}(\mathrm{BIC} 03)$. Nous en présentons une ci-dessous.

\section{Exemple}

Le problème suivant a été posé à 78 personnes dans un atelier « grand public » lors de la Fête de la Science 2000.

Deux trains avancent l'un vers l'autre sur une voie rectiligne.

Ils roulent chacun à $50 \mathrm{~km} / \mathrm{h}$.

Ils sont éloignés de $100 \mathrm{~km}$ à l'instant initial.

La mouche Cécé, qui vole à $100 \mathrm{~km} / \mathrm{h}$ part du premier train et vole vers le deuxième.

Dès qu'elle est arrivée, elle repart en sens inverse vers le premier. 


$$
\text { "antibi" — 2007/8/17 — 10:13 — page } 215 \text { — \#21 }
$$

Dès qu'elle atteint celui-ci, elle repart en sens inverse vers le deuxième, et ainsi de suite.

Quelle distance Cécé aura-t-elle parcourue au moment où elle sera écrasée dans la collision entre les deux trains?

Un élève de $5^{\text {ième }}$ a été le seul à proposer la réponse : $100 \mathrm{~km}$. (Les trains se rencontrent après une heure. Cécé ayant volé pendant une heure a parcouru 100 $\mathrm{km}^{4}$.)

Dans l'assistance il y avait des enseignants de tous les niveaux qui n'ont pas trouvé car ils ont cherché des solutions sophistiquées. Pour l'anecdote des enseignants de collège présents n'étaient pas d'accord pour accepter cette solution.

\section{Références}

[1] A. Antibi, La motivation en Mathématiques : celle du professeur? celle de l'élève?, Journées Nationales de l'APMEP, Gérardmer (1999), Bulletin APMEP n 428.

[2] J. Bichara, Les mathématiques dans le grand public et dans l'enseignement : Comparaison, Analyse Didactique, Thèse, Université Paul Sabatier, 2003.

[3] G. Brousseau, Fondements et méthodes de la Didactique des mathématiques, Recherches en didactique des mathématiques 7, no. 2 (1986), 33-116.

[4] G. Brousseau, La théorie des situations didactiques en mathématiques, La pensée sauvage, Paris, 1997.

${ }^{4}$ Puisque Cécé va deux fois plus vite que les trains, elle aura été une première fois du premier train au second quand ce dernier aura parcouru $\frac{1}{3}$ du trajet et elle $\frac{2}{3}$, c'est-à-dire $\frac{2}{3} 100$. À ce moment, les deux trains sont éloignés de $\frac{1}{3} 100 \mathrm{~km}$. Donc pour revenir du deuxième au premier, Cécé parcourra $\frac{2}{3} \frac{1}{3} 100 \mathrm{~km}$. À ce moment les deux trains sont éloignés de $\left(\frac{1}{3}\right)^{2} 100 \mathrm{~km}$. Donc pour aller une nouvelle fois du premier au second, Cécé devra parcourir $\frac{2}{3}\left(\frac{1}{3}\right)^{3} 100 \mathrm{~km}$. Et ainsi de suite. Cécé parcourt en tout :

$$
100 \frac{2}{3}\left[\frac{1}{3}+\left(\frac{1}{3}\right)^{2}+\left(\frac{1}{3}\right)^{3}+\ldots\right] \mathrm{km}=100 \frac{2}{3} \frac{\frac{1}{3}}{1-\frac{1}{3}} \mathrm{~km}=100 \mathrm{~km}
$$




$$
\text { "antibi" — 2007/8/17 — 10:13 — page 216 — \#22 }
$$

216 A. Antibi et J. Bichara : Les mathématiques dans le grand public et dans l'enseignement

ANDRÉ ANTIBI

LABORATOIRE D'ETUDES DES MÉTHODES MODERNES D'ENSEIGNEMENT L.E.M.M.E.

IREM DE TOULOUSE

FRANCE

E-mail: antibi@cict.fr

JEAN BICHARA

LABORATOIRE D'ETUDES DES MÉTHODES MODERNES D'ENSEIGNEMENT L.E.M.M.E. IREM DES ANTILLES ET DE LA GUYANE

FRANCE

(Received December, 2004) 